\title{
ESPECTROSCOPÍA DE ABSORCIÓN ATÓMICA CON LLAMA Y SU APLICACIÓN PARA LA DETERMINACIÓN DE PLOMO Y CONTROL DE PRODUCTOS COSMÉTICOS
}

\author{
FLAME ATOMIC ABSORPTION SPECTROSCOPY AND ITS APPLICATION TO THE \\ DETERMINATION OF LEAD AND CONTROL OF COSMETIC PRODUCTS \\ Wilma Gallegos ${ }^{1}$, María Vega ${ }^{1}$ y Paco Noriega ${ }^{2}$
}

\author{
${ }^{1}$ Maestría en Ciencias y Tecnologías Cosméticas, Universidad Politécnica Salesiana, Av. 12 de Octubre N24-22 y Wilson, Telf. \\ (593-2) 3962800, Quito, Ecuador. \\ ${ }^{2}$ Centro de Investigación y Valoración de la Biodiversidad, CIVABI, Universidad Politécnica Salesiana, Av. 12 de Octubre N24-22 \\ y Wilson, Telf. (593-2) 3962800, Quito, Ecuador. \\ Autor para correspondencia: mvegapi@est.ups.edu.ec
}

Manuscrito recibido el 7 de marzo de 2012. Aceptado, tras revisión, el 29 de marzo de 2012.

\section{Resumen}

La presente es una revisión bibliográfica sobre la espectroscopia de absorción atómica de llama, método sensible y confiable en la determinación de metales pesados, ya que cuenta con una energía de transmisión electrónica única para cada metal. Este hecho facilita la investigación de nuevos productos cosméticos, en los cuales la determinación de plomo ( $\mathrm{Pb}$ ) es un tema de constante interés debido a su toxicidad. Es el caso de las empresas fabricantes las cuales a más de cumplir con la reglamentación exigida por la autoridad sanitaria deben plantearse requerimientos rigurosos en las buenas prácticas de fabricación, con el afán de dar al consumidor formulaciones inteligentes que embellezcan sin repercusiones sobre la salud en el tiempo, además de la información necesaria acerca de su composición. Asimismo, la autoridad sanitaria debe verificar en la etapa de post-registro las especificaciones de cumplimiento de la normativa, controlando de una mejor manera los productos cosméticos que circulan en el país.

Palabras claves: absorción atómica de llama, plomo, cosméticos, metales pesados.

\begin{abstract}
This document is a bibliographic compilation on the flame atomic absorption spectroscopy, a simple and reliable method to determine heavy metals due to characteristic electronic transmission energy of each element. An important application is the determination of lead $(\mathrm{Pb})$ concentration in cosmetic products, because of the Pb toxicity. As a matter of fact, all the manufacture of cosmetic product have to obtain a health inspection certificate, to bring secure and trendy products to the consumers, offering not only esthetically solutions but also a chemical description of the product. The information presented is thought to give support in the process of obtaining health inspection certificates and help in the control of manufacturing of cosmetic products available in the country.
\end{abstract}

Keywords: flame atomic absorption, lead, cosmetics, heavy metals.

Forma sugerida de citar: Gallegos W., M. Vega y P. Noriega. 2012. Espectroscopía de absorción atómica con llama y su aplicación para la determinación de plomo y control de productos cosméticos. La Granja. Vol. 15(1): 18-25. ISSN: 1390-3799. 


\section{Introducción}

A lo largo del tiempo se ha logrado el desarrollo de formulaciones cosméticas inteligentes a través de la combinación exacta de cada ingrediente, permitiendo de esta manera el desarrollo de cosméticos seguros y tecnológicamente mejor elaborados. Así, los pigmentos usados como agentes colorantes por los pueblos antiguos estarían ahora prohibidos por estar compuestos por sustancias peligrosas. Por ejemplo: para el maquillaje de la cara el plomo blanco daba un color pálido, el fósforo rojo se usaba como colorante para añadir un toque de color a las mejillas y el cinabrio amarillo ( $\mathrm{HgS})$ daba brillo a los labios. Se acentuaba la intensidad de los ojos usando sombra de oropimente $\left(\mathrm{As}_{2} \mathrm{~S}_{3}\right)$ y máscara de pestañas de estibinita $\left(\mathrm{Sb}_{2} \mathrm{~S}_{3}\right)$. Es decir que estos cosméticos solían contener metales pesados como plomo, arsénico, mercurio y antimonio, los cuales, a pesar de contribuir a la belleza, representaban un riesgo para la salud.

Sin embargo, existen muchas otras investigaciones que corroboran la presencia de metales pesados en formulaciones cosméticas actuales como: tintes de cabello, maquillaje, sombras y labiales que han producido daños en el consumidor. En la presente revisión se habla del plomo presente en cosméticos y su concentración por encima de los límites establecidos en la norma (NOM-119-SSA1-1994), lo cual provocaría daños al sistema nervioso, reproductor, óseo y sería altamente carcinogénico.

Por otro lado, refiriéndonos a la Investigación y Desarrollo de productos cosméticos (I\&D), la caracterización de las materias primas constituye un punto primordial de análisis, existiendo metodologías especificas y sensibles para la determinación y cuantificación de metales pesados, una de ellas la Espectroscopía de Absorción Atómica de llama (FAAS, por sus siglas en inglés de Flame Atomic Absorption Spectrometry) en la Figura 1 se muestra el equipo utilizado. Este método está ampliamente utilizado desde hace medio siglo, y permite la detección de metales hasta en el orden de las partes por millón - ppm. La muestra por su naturaleza no puede ser analizada directamente en el equipo por lo que se la somete a un proceso previo de preparación que permite obtener una disolución del analito adecuada para la atomización. Dentro de los métodos más comunes de preparación se incluyen métodos de digestión, pudiendo ser ésta húmeda, seca o asistida por microondas. A continuación se realiza la ato- mización de la muestra ya disuelta en el FAAS, en donde los electrones de los átomos llegan a ser promovidos a orbitales más altos llegando a su estado fundamental mediante la absorción de una cantidad de energía la cual es proporcional a la concentración del elemento que se mide (Skoog et al., 2001).

De esta manera, este método espectroscópico tiene una amplia aplicación en lo que refiere a la cuantificación de metales pesados, ya que permite el análisis de prácticamente todos los elementos de la tabla periódica. Su aplicación en la investigación de nuevos productos cosméticos se aplica a: filtros solares, cremas, shampoos, productos para el cuidado de la piel, productos de higiene personal, rímel para pestañas, sombra de ojos, labiales, antitranspirantes y pasta de dientes (Salvador y Chisvert, 2007).

Sin embargo, a pesar de que en la industria cosmética la FAAS ha tenido un especial uso en el análisis de labiales, la FDA (Food an Drug Administration, por sus siglas en inglés) no determina un límite de plomo en labiales y en los cosméticos en general, lo que sí ha determinado es el límite de plomo como impureza en aditivos de colores que se ha logrado fijar en 20 ppm (FDA, 2011). Y es que el tema de la bioacumulación de plomo en el organismo ha despertado un amplio interés, ya que aunque los valores de plomo en los productos cosméticos estén dentro del límite aceptable, su uso frecuente generaría una acumulación que estaría siendo causa de intoxicación en el ser humano.

\section{Descripción del método y su aplicación para la determina- ción de plomo}

La espectroscopia de absorción atómica (AAS por sus siglas en inglés Atomic Absorption Spectroscopy), es una técnica extremadamente sensible, y especifica debido a que las lineas de absorción atómica son considerablemente estrechas (de 0,002 a 0,005 nm) y las energías de transición electrónica son únicas para cada elemento (Skoog et al., 2001). Al igual que la FAAS, la sensibilidad de la de absorción atómica por atomización de llama está en el orden de los ppm. En la industria cosmética tiene una enorme aplicación para determinaciones cualitativas y cuantitativas de metales pesados presentes en los productos, 


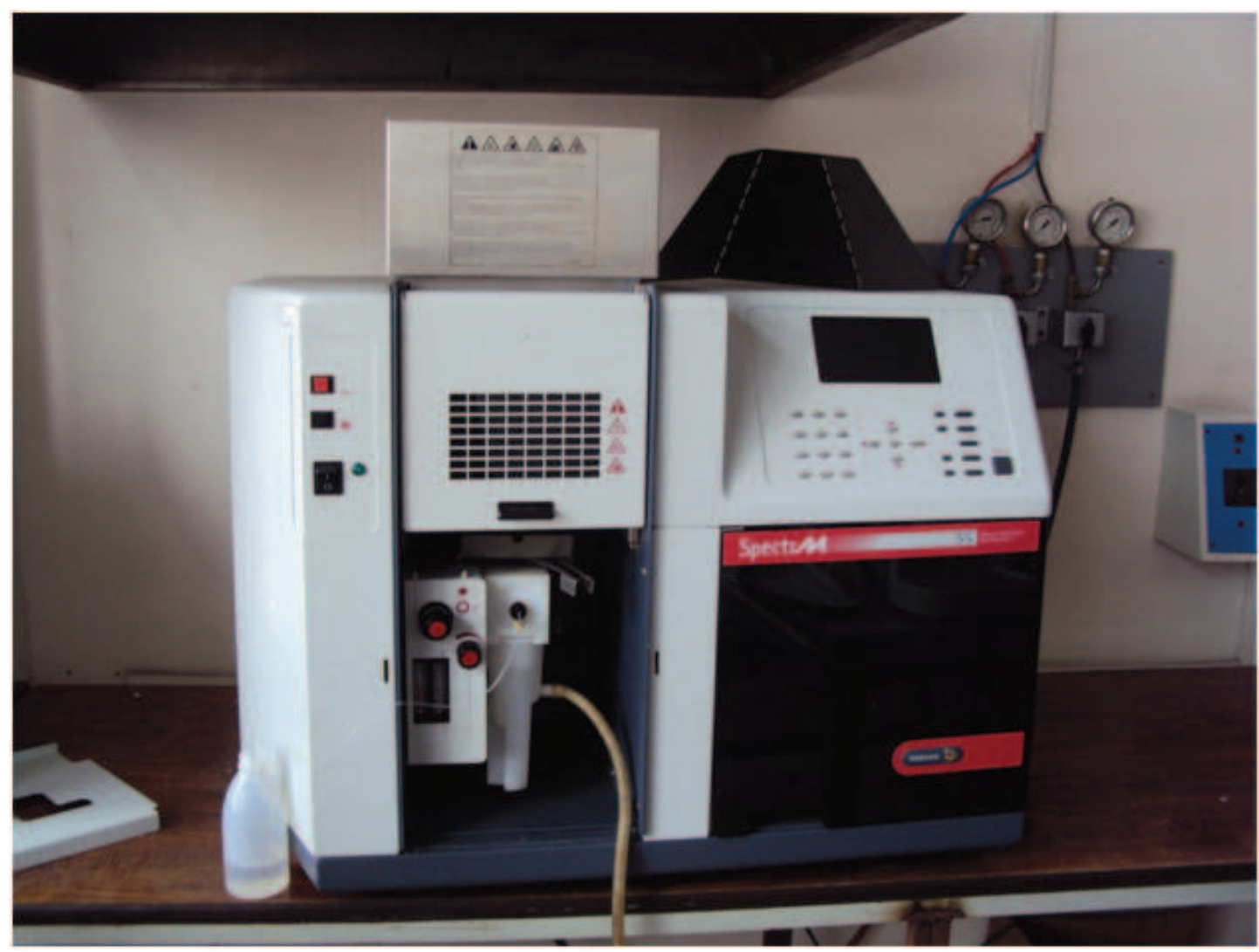

Figura 1. Equipo de Espectrometría de Absorción atómica con llama. Fuente CIVABI.

que podrían desencadenar procesos alérgicos para los consumidores o presentarse fenómenos oxidativos indeseados para el fabricante en los productos finales.

La instrumentación básica para la AA se presenta en la Figura 2, y está constituida de una fuente de radiación monocromática (específica para cada elemento a analizar), o policromática, un atomizador para producir los átomos excitados de la sustancia a analizar; un monocromador para seleccionar la longitud de onda de la radiación característica de cada elemento a analizar; un detector sensible a la radiación emitida y un procesador de la señal y de la lectura de salida.

En términos generales, el funcionamiento es el siguiente: el haz emitido por la fuente atraviesa el sistema de atomización que contiene la muestra en estado de gas atómico, ésta llega al monocromador que elimina la radiación que no interesa para el estudio, pasando así al revelador o detector de la radiación absorbida, que luego es procesada y ampli- ficada, dando como resultado una lectura de salida (AA.VV., 2007).

Existen varios artículos científicos realizados sobre la determinación de metales pesados en cosméticos empleando AAS (Oyedeji et al., 2011; Amartey et al., 2011; Chauhan et al., 2010; Sharma et al., 2011; Al-Ashban et al., 2003; Arise et al., 2010) y otros, en donde detallan específicamente la utilización de FAAS (Nnorom et al., 2005; Omolaoye et al., 2010; Peregrino et al., 2011; Hati et al., 2009; Bortoleto et al., 2004; Trejos, 2011).

Asimismo, la mayoría de autores siguen utilizando métodos tradicionales para tratar previamente a las muestras ya que las mismas deben ser introducidas en la fuente de excitación como parte de una solución. Este tratamiento (descomposición y disolución) resulta ser laborioso para obtener una muestra adecuada del analito para la atomización, consumiendo tiempo (horas) e introduciendo errores en la propia medición espectroscópica. Es por esto, que se utilizan tratamientos empleando altas 


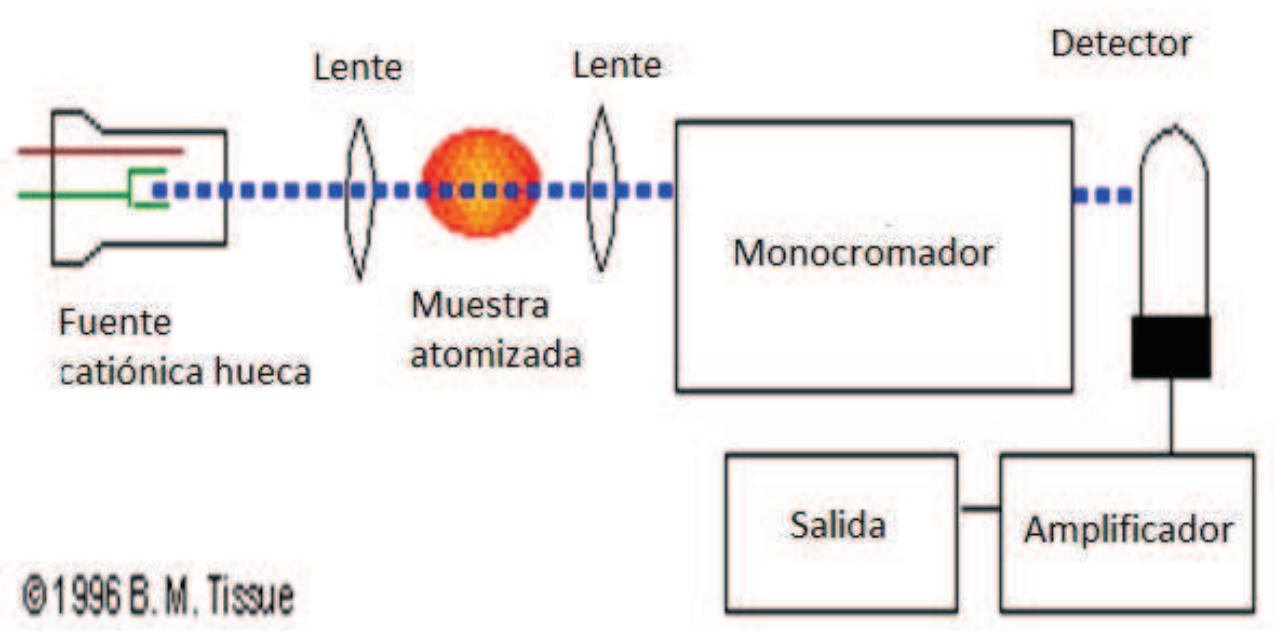

Figura 2. Esquema básico de un espectrómetro de AA. Adaptado de B M. Tissue (1996).

temperaturas a pesar de que aumenta la potencial perdida de analito por volatilización o en forma de aerosoles; también se debe cuidar el momento de adquirir los reactivos ya que el analito puede estar presente en forma de impureza en los mismos. Entre los métodos tradicionales para la descomposición y disolución de las muestras se tienen los que emplean tratamiento con ácidos minerales en caliente (mezcla de $\mathrm{HNO}_{3}$ y $\mathrm{HClO}_{4}$ ) y digestión a elevada temperatura (Skoog et al., 2001).

En cuanto al tratamiento de muestras para AAS, en la actualidad se dispone de equipos como los digestores de microondas, que ayudan a que esta tarea de tratamiento previo de la muestra no sea complejo, (Ver Figura 3) dando como resultado una preparación confiable de la muestra para realizar el análisis. Una de las razones más importantes de su uso está asociada con el ahorro de tiempo que ofrece en relación a las descritas anteriormente, también evita la perdida de elementos volátiles así como la contaminación de los vapores de ácidos en el ambiente del laboratorio, asimismo evita contaminación de las muestras al utilizar mínimas cantidades de ácido y se pueden procesar varias muestras al mismo tiempo.

Comparando las técnicas para el tratamiento de las muestras previas a su atomización en el espectrofotómetro de AA, sus diferencias son notorias. Por ejemplo las que utilizan métodos tradicionales de digestión, generalmente primero deben realizar un proceso de desecación en una estufa hasta lograr un peso constante durante 12 horas, para su posterior digestión empleando varios mililitros de $\mathrm{HNO}_{3}$ en 1 gramo de la muestra desecada hasta volver a llevarlo a la sequedad para luego repetir el procedimiento empleando $\mathrm{HClO}_{4}$ hasta la aparición de humos blancos que indican la terminación del proceso de digestión, luego se filtra y se coloca en un matraz volumétrico hasta completar el volumen con agua desionizada (Oyedeji et al., 2011; Chauhan et al., 2010; Nnorom et al., 2005; Omolaoye et al., 2010; Sharma et al., 2011; Hati et al., 2009; Arise et al., 2010); por otro lado, los métodos que hacen uso de la digestión en horno microondas, emplean menos cantidad de muestra (miligramos), al igual que ácido (1 mililitro) y el tiempo que se emplea en el procedimiento que consta de tres etapas tiene una duración total de 65 minutos (Amartey et al., 2011; Peregrino et al., 2011) evidenciándose así la utilidad de este último método.

Una vez que se obtiene la muestra lista para ser empleada en el espectrofotómetro de absorción atómica con llama, se debe elegir la longitud de onda adecuada para la identificación plomo $(\mathrm{Pb})$, que es de 217,0 nm, y para corrección de la radiación de fondo se utiliza una lámpara de deuterio. Para eliminar interferencias negativas en la técnica de absorción atómica tanto de los patrones como de las muestras se emplea EDTA 0,1 mol/L. 


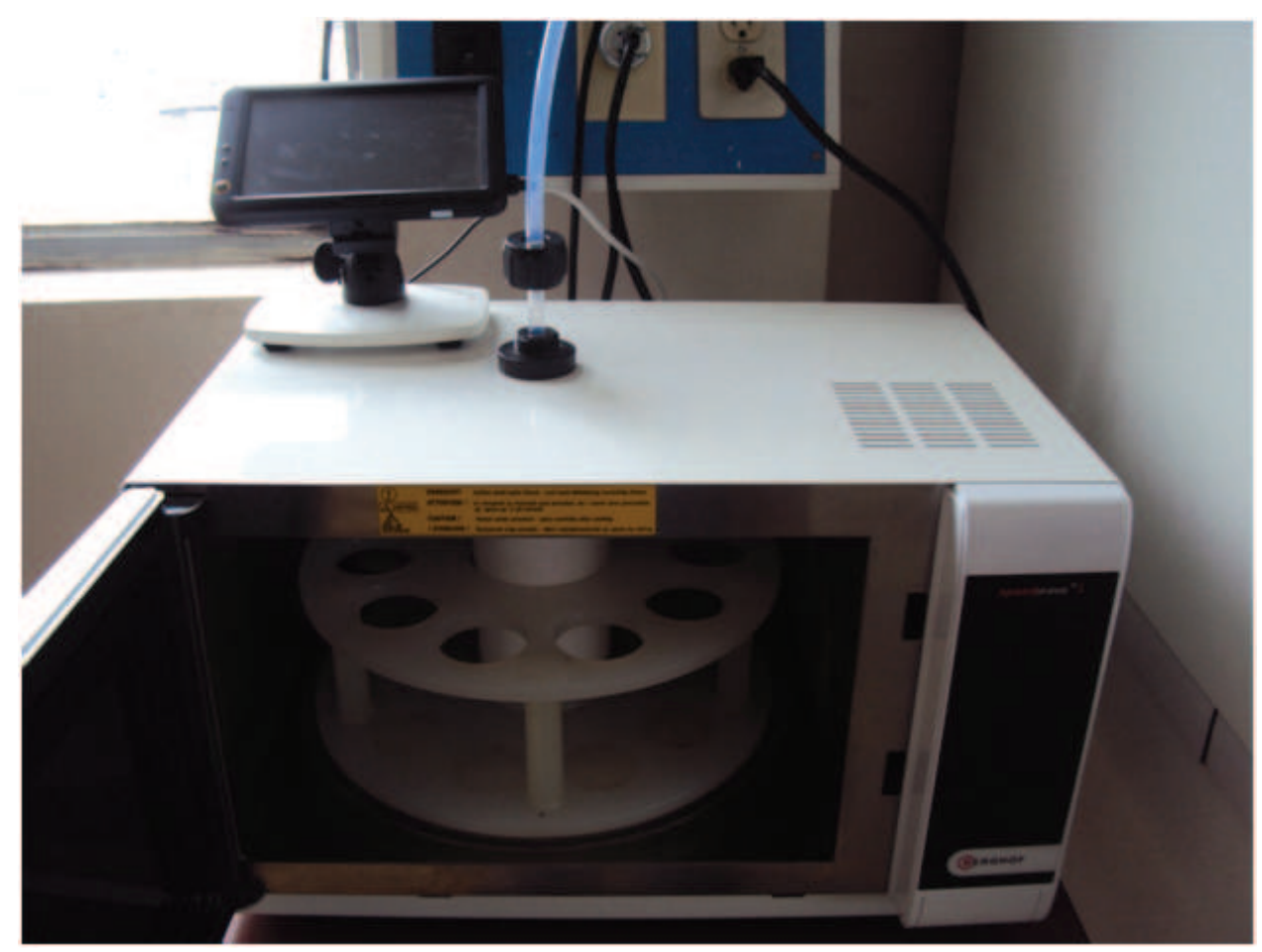

Figura 3. Digestor de microondas. Fuente CIVABI.

Posteriormente, se realiza la curva de calibración utilizando el método de dilución de estándar, el mismo que debe ser preparado periódicamente para cubrir el intervalo de concentraciones correspondientes al analito, cualquier desviación del patrón respecto a la curva de calibración original puede ser utilizada para corregir la lectura analítica, generando resultados confiables y replicables.

El tipo de llama más utilizado para la determinación de plomo en los productos cosméticos es el de la mezcla aire (oxidante) - acetileno (combustible), la cual posee una temperatura entre 2.100 a $2.400{ }^{\circ} \mathrm{C}$, siendo óptima para llevar a los átomos a su estado fundamental (Skoog et al., 2001).

\section{Presencia de plomo en la inves- tigación de nuevos productos cosméticos}

El plomo $(\mathrm{Pb})$ es un metal pesado que cuando ingresa en el organismo se acumula en los huesos. Este metal ingresa por las vías respiratorias o al ingerir- lo, pasando desapercibido. La intoxicación por plomo puede tener efectos muy serios en la salud y especialmente en los bebés en periodo de formación; el plomo en la sangre de la madre puede causar abortos espontáneos, bebés prematuros o bebés con bajo peso y también puede afectar el desarrollo cerebral del niño cuando nace. Por ello es de importancia asegurarse que futuras madres y niños pequeños no entren en contacto con productos que puedan contener $\mathrm{Pb}$. Entre estos productos se pueden identificar: pinturas, cerámicas de artesanía, latas de alimentos soldadas, agua y cosméticos.

Específicamente, en la industria cosmética un punto crítico es el análisis de las materias primas, especialmente en el caso de los colorantes y pigmentos el plomo puede hallarse como una impureza. De ahí la necesidad de asegurar que el producto cosmético sea inocuo para el consumidor al ser utilizado en condiciones normales y al mismo tiempo sea eficaz cumpliendo la función que declara en el envase, por lo que cada empresa debe ampliar sus criterios de seguridad y exigencia realizando controles de calidad de las materias primas hasta el producto terminado. 
La legislación de los Estados Unidos y europea establecen restricciones para algunos productos, $\mathrm{CO}^{-}$ mo ejemplo tenemos el acetato de plomo, en el 2007 un informe demostraba que los labiales de 33 marcas mundialmente conocidas contenían niveles de plomo. En ellos se encontró trazas toleradas por la legislación internacional que no presentaban riesgo identificado para la salud humana. Así, la empresa privada argumentó que sus productos contienen niveles bajos de contaminantes y que no son nocivos.

Sin embargo, el problema es que los consumidores están expuestos repetidamente, día tras día, a estos niveles "bajos" del metal pesado y esa reiteración genera una bioacumulación, que es lo que al final puede acarrear problemas a la salud.

Asimismo, cabe mencionar que en un estudio realizado por científicos de la FDA, se encotnró la presencia de plomo en 20 labiales de barra, con valores comprendidos entre $0,09 \mathrm{ppm}$ y 3,06 ppm, con un valor promedio de 1,07 ppm teniendo como límite de detección el valor de $0,04 \mathrm{ppm}$. Por lo que la FDA concluyó que los niveles de plomo se encuentran dentro de los rangos esperados para los labiales formulados con aditivos de colores permitidos y otros ingredientes y preparados cumpliendo las normas de buenas prácticas de fabricación (FDA U.S Food and Drug Administration, 2011).

\section{Conclusiones}

En la actualidad, las industrias cosméticas, sus departamentos de investigación realizan pruebas exhaustivas para asegurar de que los productos cosméticos no van a causar daño en el consumidor. El método de la espectrofotometría de absorción atómica de llama, es de mucha utilidad para la investigación de nuevos cosméticos que sean seguros para la salud de los consumidores, al permitir detectar y cuantificar la presencia de metales pesados en los mismos, sobre todo la investigación de plomo resulta ser un tema de continuo interés, por los riesgos tóxicos que implica este metal en el ser humano.

Es importante destacar que el método de Absorción Atómica de llama es ampliamente utilizado para realizar investigaciones científicas, que en el área cosmética están enfocadas a la determinación de metales pesados, tanto por su especificidad, sensibilidad y costo del equipo. Dado que esta tecnología se encuentra disponible en Ecuador resul- ta interesante plantear la realización de estudios para analizar los productos que se tienen en el mercado sean estos nacionales o importados. Estudios que deberían constituirse en un tema de análisis con las instituciones reguladoras al momento de obtener una Notificación Sanitaria para un producto cosmético.

En general, la mayoría de investigaciones para determinar los valores de plomo como impurezas de los aditivos de colores concluyen que casi todos los productos cosméticos testeados están dentro de los valores normales; pero aún es un tema de interés entre la comunidad científica estudiar los efectos de la bioacumulación de este metal en los seres humanos, a largo plazo.

\section{Referencias}

AA.VV. 2007. Manuale del Cosmetologo. Tecniche Nuove, Italia, ricerca applicata, Progettazione, Engineering, Produzione, Marketing, Packaging, Discipline collegate.

Al-Ashban, R., M. Salam y A. Shah. 2003. Kohl (surma): a toxic traditional eye cosmetic study in saudi arabia. Journal of the Royal Institute of Public Health, 118: 292-298, URL 〈www.elsevierhealth.com/journals/pubh〉.

Al-Saleh, I., S. Al-Enazi y N. Shinwari. 2009. Assesment of lead in cosmetic products. Regul. Toxicol. Pharmacol., 54(2): 105-113, URL 〈http://www.ncbi.nlm.nih.gov/pubmed /19250956〉.

Amartey, E., A. Ssumadu-Sakyi, C. Adjei, F. Quashie, G. Duodu y N. Bentil. 2011. Determination of heavy metals concentration in hair pomades on teh ghanaian market using atomic absorption spectrometry technique. British Journal of Pharmacology and Toxicology, 2(4): 192-198.

Arise, R., A. Tella, A. Igunnu y S. Malomo. 2010. Elemental analysis of local galena based eyeliner and its effects on some enzymes of rat liver and brain. International Research Journal of Biotechnology, 1(4): 059-064, URL 〈www.interesjournals.org/IRJOB.

Barlas, N., M. Irget y M. Tepecik. 2011. Mineral content of the rocket plant (eruca sativa). Afri- 
can Journal of Biotechnology, 10(64): 14080-14082, URL $\langle$ www.academicjournals.org/ AJB $\rangle$.

Bortoleto, G., G. Macarovscha y S. Cadore. 2004. Determination of cadmium by flame-atomic absorption spectrometry after preconcentration on silica gel modified with cupferron. J. Braz. Chem. Soc., 15(2): 313-317.

CancerHelp UK. Lead in lipstick causing cancer. URL 〈http://cancerhelp.cancerresearchuk.org /about-cancer/cancer-questions/lead-in-lipstick -causing-cancer $\rangle$.

Chauhan, A., R. Bhadauria, A. Singh, S. Lodhi, D. Chaturvedi y V. Tomar. 2010. Determination of lead and cadmium in cosmetic products. Journal of Chemical and Pharmaceutical Research, 2(6): 92-97.

Ciaralli, L., R. Giordano, S. Cassoma, A. Sepe y S. Costantini. 1996. Determination of chromium and nickel in comercial foam bath products by eta-aas. Ann. Ist. Super. Sanita, 32(3): 381-385.

Das, S., A. Grewal y M. Banerjee. 2011. A brief review: Heavy metal and their analysis. International Journal of Pharmaceutical Sciences Review and Research., 11(1), URL 〈www.global researchonline.net.)

Envionmental defence. 2011. Heavy metal hazard. the health risks of hidden heavy metals in face makeup. URL 〈http:/ / environmentaldefence.ca/sites/default /files/report_files/HeavyMetalHazard \%20FINAL.pdf $\rangle$.

FDA U.S. Food and Drug Administration. 2010. Color additives permitted for use in cosmetics. URL 〈http://www.fda.gov/Cosmetics /GuidanceComplianceRegulatoryInformation

/VoluntaryCosmeticsRegistrationProgramVCRP /OnlineRegistration /ucm109084 .

FDA U.S Food and Drug Administration. 2011. Lipstick and lead: Questions and answers. URL 〈http://www.fda.gov/cosmetics /productandingredientsafety

/productinformation/ucm137224.htm $\rangle$.

Hall, B., S. Tozer, M. Coroama, W. Stelling, M. Leneveu-Duchemin, C. McNamara y M. Gibney. 2007. European consumer exporsure to cosmetic products, a framework for conducting population exposure assessments. Food Chem Toxicol., 45(11): 2097-2108, URL 〈http://www.ncbi.nlm.nih.gov/pubmed /17683841〉.

Hati, S., G. Dimari y V. Ogugbuaja. 2009. Levels of potentially toxic metals in synthetic industrial essential oils utilized in northern nigeria. The Pacific Journal of Science and Technology, 10(1), URL 〈www.akamaiuniversity.us/PJST.htm $\rangle$.

Health Canada, Consumer Product Safety. 2011. Draft guiadance on heavy metal impurities in cosmetics. URL 〈http://www.hc-sc.gc.ca/cps-spc/legislation /consultation/_cosmet/metal-metaux-consult -eng.php $\rangle$.

Hepp, N., W. Mindak y J. Cheng. 2009. Determination of total lead in lipstick: development and validation of a microwave-assisted digestion, inductively coupled plasma-mass spectrometric method. J. Cosmet. Scie., 60(4): 405-414, URL 〈http://www.ncbi.nlm.nih.gov/pubmed /19691936〉.

LifeExtension. 2011. Heavy metal toxicity. URL 〈http://www.lef.org/protocols/prtcls-txt /t-prtcl-156 .

Nnorom, I., J. Igwe y C. Oji-Nnorom. 2005. Trace metal contents of facial (make-up) cosmetics commonly used in nigeria. African Journal of Biotechnology, 4(10): 1133-1138.

Omolaoye, J., A. Uzairu y C. Gimba. 2010. Heavy metal assessment of some eye shadow products imported into nigeria from china. Archives of Applied Science Research, 2(5): 76-84, URL $\langle$ www.scholarsresearchlibrary.com $\rangle$.

Oyedeji, F., G. Hassan y B. Adeleke. 2011. Hydroquinone and heavy metals levels in cosmetics market in niegeria. Trends in Applied Sciences Research, 6(7): 622-639.

Peregrino, C., M. Moreno, S. Miranda, A. Rubio y L. Leal. 2011. Mercury levels in locally manufactured mexican skin-lightening creams. Int. J. Environ. Res. Public Health, 8: 2516-2523, URL 〈www.mdpi.com/journal/ijerph〉.

Revista Salud Alternativa. 2011. Plomo en lápices labiales: Riesgos maquillados para la salud. 
URL 〈http://www.revistasaludalternativa.com /plomo-en-lapices-labiales-riesgos-maquillados -para-la-salud/ $\rangle$.

Sainio, E., R. Jolanki, E. Hakala y L. Kanerva. 2000. Metals and arsenic in eye shadow. Contact Dermatitis, 42(1): 5-10.

Salvador, A. y A. Chisvert. 2007. Analysis of Cosmetic Products. Elsevier, Amsterdam.

Sharma, D., S. Rai, S. Arora, P. Gupta, R. Sharma y A. Chopra. 2011. Study of the trace elements in Aloe vera L. (Aloe barbandensis Miller) viz. Liliaceae and its biological and environmental Importance. J. Chem. Pharm. Res., 3(3): 64-68, URL $\langle$ www.jocpr.com $\rangle$.

Skoog, D., F. Holler y T. Nieman. 2001. Principios de Analisis Instrumental. Mc Graw Hill/Interamericana de España, S.A.U, 5 edición.
Trejos, S. 2011. Validación de la metodología para la determinación cuantitativa de plomo en tintes cosméticos disponible en el mercado nacional por espectroscopia de absorción atómica con llama. Tecnología en Marcha, 21(3): 42-46.

Vejledning fra Miljostyrelsen. 2000. Guideline on safety assessment of cosmetic products. Product information/dossier on cosmetic products, in the Danish Order of Cosmetic Products. Environmental Guideline, 10, URL 〈http://www2.mst.dk/udgiv/publications /2000/87-7944-336-2/pdf /87-7944-337-0.pdf $\rangle$.

Womens Health. 2011. Cosmetics and your health. URL /http://www.womenshealth.gov /publications/our-publications/fact-sheet /cosmetics-your-health.pdf $\rangle$. 